\title{
Multi-therapies in androgenetic alopecia: review and clinical experiences
}

\author{
Alfredo Rossi*, Alessia Anzalone*, Maria Caterina Fortuna*, \\ Gemma Caro*, Valentina Garelli*, Giulia Pranteda*, \\ \& Marta Carlesimo ${ }^{\dagger}$ \\ *Department of Internal Medicine and Medical Specialties, "Sapienza" \\ University of Rome, Italy and †Dermatology "Sapienza" University of Rome, \\ II School, Italy
}

\begin{abstract}
Androgenetic alopecia (AGA) is a genetically determined progressive hair-loss condition which represents the most common cause of hair loss in men. The use of the medical term androgenetic alopecia reflects current knowledge about the important role of androgens and genetic factors in its etiology. In addition to androgen-dependent changes in the hair cycle, sustained microscopic follicular inflammation contributes to its onset. Furthermore, Prostaglandins have been demonstrated to have the ability in modulating hair follicle cycle; in particular, PGD2 inhibits hair growth while PGE2/F2a promote growth. Due to the progressive nature of AGA, the treatment should be started early and continued indefinitely, since the benefit will not be maintained upon ceasing therapy. To date, only two therapeutic agents have been approved by the Food and Drug Administration and European Medicines Agency for the treatment of AGA: topical minoxidil and oral finasteride. Considering the many pathogenetic mechanisms involved in AGA, various treatment options are available: topical and systemic drugs may be used and the choice depends on various factors including grading of AGA, patients' pathological conditions, practicability, costs and risks. So, the treatment for AGA should be based on personalized therapy and targeted at the different pathophysiological aspects of AGA.
\end{abstract}

KEYWORDS: alopecia, dermatopathology, therapy-topical

\section{Introduction}

Androgenetic alopecia (AGA) is a genetically determined progressive hair-loss condition which represents the most common cause of hair loss in men. The incidence and severity is

Address correspondence and reprint requests to: Alessia Anzalone, Section of Dermatology, Department of Internal Medicine and Medical Specialties, University "La Sapienza”, Rome, Viale del Policlinico, 155, 00161, Rome, Italy, or email: alessia.anzalone@uniroma1.it.

Conflict of Interest: No conflict of interest. more common in Caucasian men than other nationalities (1). It is estimated that prevalence rates in Caucasian populations is around $12 \%$ among men aged 18-29 years and approximately $50 \%$ among men aged $40-49$ years; by the age of 80 , over $90 \%$ of Caucasian men are affected to a various degree (2). AGA is characterized by progressive miniaturization of the hair follicle, resulting from alteration in the hair cycle; in fact the duration of the anagen phase gradually decreases, whilst the length of telogen remains constant or is prolonged. This results in a 
reduction of hair in anagen and a relative increase in numbers of follicles in telogen (3). There is also a progressive miniaturization of the entire follicular apparatus, leading to gradual conversion of terminal hairs into vellus hairs with a progressive reduction in hair density. The pattern of loss follows the scale developed by Hamilton (2), and later extended by Norwood (4) which defines seven distinct categories. Three areas of the scalp are affected preferentially: the temples, vertex and mid frontal scalp, whereas the occipital region is spared. This typical pattern is due to the fact that hair follicles in the frontal and vertex regions are androgen-sensitive thanks to the elevated expression of androgen receptors (ARs), whereas the occipital and temporal regions contain androgen-insensitive hair follicles (5).

The use of the medical term androgenetic alopecia reflects current knowledge about the important role of androgens and genetic factors in AGA etiology. It has long been known that the presence of testosterone in hair follicles is a prerequisite for AGA (6), while genetic factors modify the magnitude of the hair follicle response to circulating androgens (7). In consideration of the wide range of expressed phenotypes in AGA, a polygenic mode of inheritance is established. Gene association studies highlighted that the AR gene variants are the primary culprit responsible for AGA development. The ARs determines the sensitivity of hair follicle to androgens and polymorphism of the AR gene is associated with AGA; the Stu 1 polymorphism has the most significant association (8). Several other genes that could be associated include $5 \alpha$ reductase, aromatase, estrogen receptor $\alpha$ and IGF-2 genes (9).

The role of androgen in AGA is well established (1). The circulating testosterone is converted by 5a-reductase enzyme into 5adihydrotestosterone (DHT), which has tenfold higher affinity to AR compared to testosterone. The predisposed scalp exhibits high levels of DHT with an increased expression of the ARs (10). The cross-talk between the dermal papillae and the hair follicle cells unfolded under the influence of androgens results from the secretion of many factors from the dermal papillae which causes premature termination of anagen and premature entry into catagen. Catagen occurs as a consequence of decreased expression of anagen maintaining factors, such as the growth factors IGF-1, bFGF and VEGF (11). Furthermore, an increased expression of cytokines, such as $\operatorname{TGF} \beta 1$, IL $-1 \alpha$ and TNF $\alpha$ promotes apoptosis.
In addition to androgen-dependent changes in the hair cycle, sustained microscopic follicular inflammation is considered a co-factor in the etiology of AGA. A modest degree of chronic inflammation around the upper part of hair follicles has been described by many investigators (12). The observation of activated T-cells infiltrating about the lower portions of follicular infundibulum suggests that the primary causal event for the triggering of inflammation might occur near the infundibulum (13). Micro inflammation may be triggered by resident microbial flora in the case of seborrhea, toxins and oxidative stress; other factors include the aging process, smoking, UV, and environmental pollutants. These stressful influences lead to the formation of free radicals which damage the hair follicles.

An outline is emerging for a fundamental role of prostaglandins (PGs) in modulating hair follicle cycle; in particular, PGs D2, E2, and F2a have the ability to regulate hair growth. Clinical data have demonstrated that PGD2 inhibits hair growth while PGE2/F2a promote growth $(14,15)$. Recent studies suggested that PGD2 level is elevated in male balding scalp (14), whereas PGE2 has been shown to be reduced.

PGD2 is synthesized by prostaglandin D2 synthase (PTGDS), an enzyme hormone responsive expressed in the outer root sheath inferior to the bulge. PGD2 has two known receptors, GPR44 and PTGDR (16). GPR44 was found to be necessary for the decrease in hair growth by PGD2; its binding to the GPR44 receptor expressed in the hair follicle seemed to induce miniaturization of the follicle, thus probably, preventing the maturation of stem cells into progenitor cells and of vellus-like hair into terminal hair. This knowledges could be important for the creation of new target therapies for AGA, which inhibit the activity of PTGDS, PGD2 or GPR44 (16).

Thus the exact pathogenetic mechanism underling AGA is not well elucidated, further studies are needed to better understand it.

\section{Management}

Although AGA is a paraphysiological phenomenon, it causes significant changes in the outward appearance of the patients and, consequently, deep social implications particularly in younger men (1). Due to the progressive nature of AGA, treatments should be early started and indefinitely continued, since the 
benefit will not be maintained upon ceasing therapy.

Considering the many pathogenic mechanisms involved in the onset of AGA, a various treatment options are available. Topical and systemic drugs, individually or combined, may be used and the choice depends on various factors including grading of AGA, efficacy, practicability, costs and risks (17).

To date only two therapeutic agents have been approved by the Food and Drug Administration (FDA) and European Medicines Agency (EMA) for treatment of AGA: topical minoxidil and oral finasteride ( $1 \mathrm{mg} /$ day) (5).

\section{Medical management}

\section{Minoxidil}

Minoxidil solution is the most frequently used drug for topical application (18)] and it is strongly recommended as a first-line topical treatment for men with mild or moderate AGA (19).

The mechanism by which minoxidil promotes hair growth is still not completely understood. It is converted to minoxidil sulphate, an active metabolite which opens ATP-sensitive potassium channels localized on the smooth muscular cells of the peripheral artery, leading to a vasodilatory effect. It is been observed that minoxidil is able to increase the m-RNA expression for the VEGF in the dermal papilla, inducing angiogenesis (20). It has been shown that minoxidil stimulated PGE2 and leukotriene B4 in vitro production by dermal papilla cells, whereas inhibited prostacyclin synthesis. In addition, it increases the expression of one of the four PGE2 receptors called EP2 which may contribute to anagen prolongation. Minoxidil promotes also the survival of dermal papilla cells by increasing Bcl-2/Bax ratio and by activating ERK and Akt. It was postulated that minoxidil would have antifibrotic properties by inhibiting activity of the enzyme lysyl hydroxylase in human skin fibroblast leading to synthesis of a collagen deficient in hydroxylysine (21).

Progressively, thanks to minoxidil, the miniaturized hair follicle stay longer in the anagen phase, producing hair similar to the terminal hair and decreasing shedding (21).

In approximately $50 \%$ men using minoxidil solution, the hair loss process is arrested by minoxidil; in addition, a small percentage of men experiences mild to moderate degrees of hair regrowth. However, after discontinuing the therapy hairs fall out again (18).

Minoxidil solution contains ethanol, water, and propylene glycol as vehicle. It is available in $2 \%$ and $5 \%$ solutions; the $5 \%$ minoxidil has shown higher efficacy than the $2 \%$ solution (22). Minoxidil have to be applied twice a day with the dosage of $1 \mathrm{~mL}$; 4-6 months of treatment are needed to see significant results and the maximal effect is reached after approximately 1 year. Patients answer better to treatment if hair miniaturization is not advanced (Hamilton $<$ IV) (18).

The safety profile of topical minoxidil solution is favorable and its most common side effects are scalp pruritus and scaling due to irritant and allergic contact dermatitis. The $5 \%$ minoxidil formulation, which contains more propylene glycol was usually associated with a higher number of cases of itching, erythema, and dryness (1). A common side effect due to the action of Minoxidil is telogen effluvium, caused by the encouragement of hairs already in the telogen phase to shed early (17). The use of topical minoxidil can also induce hypertrichosis in different areas out of the scalp because of local/systemic absorption or possible contamination of them (22). Patient's compliance is thought to be the major limiting factor (23).

Minoxidil could be associated with other active principles, separately administred or using a galenic lotion. Combinations containing minoxidil-pyrithione zinc and minoxidilhydrocortisone have been shown to improve hair growth compared with the use of minoxidil alone.

\section{Finasteride}

Finasteride, a synthetic 4-aza-3-oxosteroid compound with poor aqueous solubility, is a highly selective inhibitor of type II 5 alpha reductase. Binding irreversibly to the enzyme, it inhibits the conversion of testosterone to DHT decreasing serum and cutaneous DHT concentrations (24). The underlying principle for its use in the treatment of AGA is the reduction of DHT production and the inhibition of androgen-dependent miniaturization of hair follicles. The clinical effectiveness and safety of finasteride has been evaluated in a well controlled clinical trials which monitored 1.879 men with AGA, demonstrating that treatment with oral finasteride $1 \mathrm{mg} / \mathrm{d}$ for 5 years resulted in hair loss reduction. In order to evaluate the long term efficacy and safety of oral finasteride $1 \mathrm{mg} / \mathrm{d}$, we enrolled 118 men aged 
between 20 and 61 years affected by AGA and monitored for a 10 years period. The results underlined that finasteride $1 \mathrm{mg} / \mathrm{d}$ administration produced significant and durable increases in hair growth (25). Of the 113 patients followed for 10 years, only $14 \%$ worsened, while the remaining had benefits $(21 \%)$ from the treatment duration or persisted in their improvements (65\%). In our opinion, the result after the first year can help in predicting the effectiveness of the treatment. In fact, the majority of patients that do not show any improvement within the first year of treatment could be considered not respondent to a long-term therapy (25).

Finasteride $1 \mathrm{mg} / \mathrm{d}$ is generally well tolerated; the reported sexual side effects are few and not age related (26) and appear to resolve on cessation of the drug $(26,27)$. The increased estrogen levels, caused by partial conversion of testosterone in estradiol through the aromatase enzyme, can lead to problems like water retention, adiposity, gynecomastia and increased risk of breast cancer, while low levels of DHT can lead to sexual dysfunctions, such as loss of libido, erective dysfunction and reduction in amount of ejaculated semen (26). According to a very large prospective study, the effect of finasteride on sexual functioning is minimal for most men but may worsen the patient's quality of life or even reduce compliance to treatment. The satisfactory clinical results and the few side effects observed, led to consider finasteride an effective treatment (25).

The FDA has approved the use of finasteride in oral formulations, but considering that the oral administration has led to undesirable systemic side effects and that 5a-reductase is mainly located in the hair follicle, the use of a percutaneous approach was investigated by several authors $(27,28)$.

A study that evaluated a finasteride $0.25 \%$ topical solution in comparison to the oral finasteride $1 \mathrm{mg} / \mathrm{d}$, found a strong and similar inhibition of plasma DHT after 1 week of treatment with the topical and tablet finasteride formulations, while finasteride plasma exposure was significantly lower with the topical than with the oral formulation (28).

A double blind, randomized clinical trial of 45 male baldness patients that compared the therapeutic effects of finasteride gel $1 \%$ and the oral finasteride $1 \mathrm{mg} / \mathrm{d}$ after 6 months of treatment proved with serial measurement a significant increase in terminal hair counts in both groups and showed no significant statistical differences between two groups (29). Preliminary results of our study evaluating the efficacy of finasteride $0.5 \%$ topical solution in treatment of AGA have shown that the application of a $1 \mathrm{~mL} / \mathrm{d}$ induced a clinical improvement in absence of side effects. The results of these studies demonstrated that topical finasteride represents a good replacement of oral therapy, especially in patients who worry about its side effects. However, further studies are needed to confirm its efficacy and the long term side effects.

\section{Other 5-alpha-reductase inhibitors}

Dutasteride, a dual inhibitor of both type I and type II 5 alpha-reductase, has recently been tested to improved efficacy over finasteride in promoting hair growth (30). Type II 5a-reductase is the predominant 5a-reductase enzyme expressed in scalp, while type I 5a-reductase is principally expressed in scalp sebaceous glands. Dutasteride is approximately 3 times more potent than finasteride at inhibiting type II 5 alpha reductase and 100 times more potent at inhibiting of the type I. A multicenter, prospective study in which 110 Japanese male patients with AGA took dutasteride at the dose of $0.50 .5 \mathrm{mg} / \mathrm{d}$ for 52 weeks, determined long-term safety, tolerability and efficacy of dutasteride within this study population (31). So, dustasteride could be considered as a potential treatment option for AGA; furthermore, for its effects on sebaceous glands, it could be useful also in the treatment of patients with AGA associated with seborreic dermatitis.

As in alternative to oral finasteride, Serenoa repens could be considered an approach in treating low or moderate AGA (19). Serenoa repens is a plant of the Arecaceae's family and its berries are highly enriched with fatty acids, phytosterols and flavonoid which have antiandrogenic activity through the competitive and nonselective inhibition of 5-alpha reductase type I and II $(32,33)$. Inhibiting 5aR, it decreases DHT uptake by hair follicle and the binding of DHT to ARs. Furthermore, Serenoa activates the estrogens receptor contributing to the anagen maintenance and to the catagen normalization. In literature there are few articles that underline the effectiveness of Serenoa in treating AGA. In a small study of 10 males with AGA taking oral Serenoa, improvement was seen in $60 \%$ (32). In our study (33), we compared the therapeutic effect of Serenoa with finasteride. We enrolled 100 patients with mild and moderate AGA; one group received Serenoa $320 \mathrm{mg}$ every day for 24 
months while the other received finasteride $1 \mathrm{mg}$ for the same period. The results showed that patients with an increase in hair growth are $38 \%$ in the group treated with Serenoa and $68 \%$ in the second group. We also observed that Serenoa acts prevalently in the vertex, while finasteride acts in both the front area and the vertex (33). The most common side effects is mild stomach discomfort which can be alleviated by taking it after food. So, with the recommended dose of $320 \mathrm{mg} /$ day, Serenoa, for its uncommon side effects is considered a non invasive approach to AGA.

\section{Other treatments}

Considering the multifactorial etiology of AGA, besides the treatment with minoxidil and finasteride and, more successfully, hair transplantation, other active principles were investigated.

\section{Topical antiandrogen}

Knowledge of the important role of androgen in genetically predisposed individuals leads to the idea that the use of AR blockers may be useful in the treatment of AGA (7). However, systemic anti-androgen treatments cannot be used in men due to its feminization effects, such as gynecomastia, feminization and impotence. For this reason it is necessary to use AR blockers that block AR selectively in scalp hair follicles. Recently, fluridil, a topical antiandrogen similar in structure to flutamide has been developed for use in AGA (1). It suppresses ARs in hair follicles and degrades into inactive metabolites without systemic anti-androgenic effects. A double-blind, placebo-controlled study showed that patients using topical fluridil had an increase in the anagen to telogen ratio, and the maximum attainable effect was achieved within the first 90 days of daily use. Sexual function, libido, hematology and blood chemistry values were normal over the duration of the investigation (1). Nevertheless a long term study is required to further investigate fluridil's long-term safety and effectiveness in AGA.

\section{Topical ketoconazole}

There is some evidence that ketoconazole, an imidazole broad-spectrum antifungal and steroid biosynthesis inhibitor, when used topically could stimulate hair growth. The mechanism is unknown, but may involve reducing inflammation through its anti-inflammatory effects against
$\mathrm{T}$ cells and through its activity against microflora of the skin by Malassezia (34). It also has antiandrogenic properties through DHT pathway disruption.

So, Ketoconazole shampoo, for its antiinfiammatory and anti-androgenic properties, is a good additive treatment and could be useful in case of associated seborrheic dermatitis.

However, clinical trials are needed to evaluate the effectiveness of ketoconazole in the treatment of AGA (1).

\section{Prostaglandin F2 $\alpha$ analogues}

Prostaglandins (PGs) have already been shown to be important in hair follicle function $(14,16)$. Consequently, PGs dysregulation may contribute to AGA pathogenesis, offering potential therapeutic targets for its treatment. Bimatoprost and latanoprost, two analogues of PG F2a used to decrease ocular pressure in glaucoma, were incidentally noted to cause hair lengthening (15). This clinical observation led to the FDA approval of the use of bimatoprost $0.03 \%$ solution for hypotrichosis of the eyelashes (35). The discovery of the potency for latanoprost and bimatoprost to induce hair lengthening remains some of the strongest evidence to implicate PGs in hair follicle function. The PG analogues stimulate hair growth supposedly by prolonging the anagen phase of the hair cycle (35). As previously mentioned, one proposed action of minoxidil that may be relevant to hair regrowth is stimulation of PG synthesis (21). So, it would seem sensible to consider the direct application of a PG analogue to the hair follicle, rather than to indirectly stimulate PG synthesis with minoxidil (14). However, further studies are needed to confirm its long term efficacy and side effects.

\section{Nigella sativa}

Nigella sativa (NS) is an annual flowering plant native to Mediterranean countries, Pakistan and India with a wide spectrum of pharmacological actions. NS seeds and its oil extract have been demonstrated to have anti-tumoral, anti-oxidant and anti- inflammatory activities (36). Thymoquinone, which represents the predominant component (30-48\%), showed anti-inflammatory effects for its ability to suppress proinflammatory cytokines such as IL-1b, TNF-a, IL-4, IL-5, IL-3; furthermore it inhibits COX-2 expression, PGD2 synthesis and NF-kB activation, contributing to the regulation of hair cycle 
(36). Regarding this, we could consider the use of NS as a possible treatment for AGA. Our double-blind, placebo controlled and randomized study in which ten patients affected by telogen effluvium were treated with $2 \mathrm{~mL}$ of topic formulation containing $0.5 \%$ NS essential oil, $3 \%$ glycerin, $0.4 \%$ lavender essential oil and $60 \%$ alcohol to $100 \mathrm{~mL}$ every day for 3 months, demonstrated NS efficacy by an improvement of hair density and hair thickness after 3 months of treatment and a reduction of inflammation observed in the majority of patients (37).

However clinical trials are needed to evaluate the effectiveness of Nigella sativa in the treatment of AGA.

\section{Growth factors}

The growth and development of hair follicles is influenced by a number of different growth factors and cytokines. In this view, platelet rich plasma (PRP) isolated from whole blood can be used for its growth factors and stimulatory mediators. PRP is defined as an autologous concentration of platelets (usually more than 1,000,000 platelets $/ \mu \mathrm{L}$ ) in concentrated plasma (38); it is produced using different methods for platelet concentration through centrifugation and cell separation, and then, it is injected in the androgen-related areas of scalp. PRP is known to carry more than 20 GFs and other protein molecules, such as adhesion molecules, chemokines, which interact to promote angiogenesis, extracellular matrix remodeling, cell proliferation and differentiation (39). Main GFs stored in $\alpha$ granules of platelets are platelet- derived growth factor (PDGF), transforming growth factor-b (TGF-b) and vascular endothelial growth factor (VEGF) with their isoforms; PDGF and VEGF appear to be important mediators of hair follicle growth by increasing the perifollicular vascular plexus. GFs appear to act in the bulge area of the follicle, in which they bind to their respective receptors located in primitive stem cells of ectodermal origin, giving origin to epidermal cells and sebaceous glands (39). Furthermore, activated PRP seems to promote differentiation of stem cells into hair follicle cells through the upregulation of b-catenin, strongly expressed in the bulge region, and to prolong anagen phase through increased expression of fibroblast growth factor-7. A clinical study conducted by V. Cervelli et al suggested that the injection of PRP preparations has a positive therapeutic effect on hair loss without major side effects (40). In our experience, PRP associated with medical therapy allows to obtain clinical response in less time than the medical therapy alone. So, PRP available alone or in association with conventional drugs for AGA could constitute a useful tool for AGA treatment, however few trials in its support have been published. More randomized, controlled, double-blind studies with larger sample size, long follow-up and objective evaluation methods are needed.

\section{Laser treatments}

Paradoxical hair growth after using lasers for hair removal has triggered interest in using these devices in the treatment of AGA (24). In recent years, low-level laser therapy (LLLT), has been promoted to stimulate hair growth in patients with AGA. There is evidence that laser light can stimulate hair growth at some wavelengths, however the biological mechanism has not been defined (1). Light of 650-900 $\mathrm{nm}$ wavelengths at $5 \mathrm{~mW}$ has been suggested as an effective option for AGA (41). Leavitt et al. reported that the treatment with HairMax LaserComb was effective in increasing terminal hair density after 26 weeks of treatment; treatment group showed a significantly greater increase in mean terminal hair density than subjects in the sham device group (41). Though there has been only one published study supporting the efficacy, the device has since been approved for treating AGA. In addiction, Kim et al. reported that after 24 weeks of treatment, a low-level light therapy group showed significantly greater hair density than the sham device group (42). A statistically significant positive trend in hair growth was also observed from a multicenter, randomized, d ouble-blind study that evalueted the efficacy of the novel cold X5 hairlaser device for treating AGA (43). Further clinical trials are needed to define the efficacy of laser treatments.

\section{Potential future treatments}

\section{Cetirizina}

It is been demonstrated that PGD2 functionally inhibits hair growth through its binding to the GPR44 receptor expressed in the hair follicle and it is elevated in male balding scalp (14); so, its inhibition could be considered as a target therapy in AGA. Cetirizine is a $\mathrm{H} 1$ antihistamine and was first described as a specific anti-H1 molecule displaying potent anti allergic activity. 
However, the action of cetirizine does not apply only at the level of $\mathrm{H} 1$ receptors. A study of Charlesworth et al., investigating the effects of cetirizine on mast cell-mediator release and cellular traffic during the cutaneous late-phase reaction, showed that the production of PGD2 was clearly reduced by cetirizine treatment (44). A study of Roch-Arveiller et al., investigating the effect exerted by various concentrations of cetirizine (0.1-10 micrograms $/ \mathrm{mL}$ ) applied in vitro to human monocytes, showed that cetirizine enhanced PGE2 release by human monocytes (45).Considering the action of cetirizine in inhibiting the release of PGD2 and in stimulating of the release of PGE2, we evaluated the efficacy of galenic solution containing cetirizine $1 \%$ in the treatment of mild and moderate AGA. Our clinical experience has shown that the application of a $1 \mathrm{~mL} / \mathrm{d}$ of cetirizine topical $1 \%$ induced a clinical improvement in absence of side effects. So, cetirizine could be considered in alternative to conventional treatment of AGA, especially in case of inflammation of the scalp. However, further research and clinical trials are required to determine its long term efficacy and side effects.

\section{Adipose derived stem cells}

Recent research suggested an interplay between hair follicle cells and adipocytes, showing the potential roles of intradermal adipocytes in hair follicle thanks to their regulatory properties during hair follicle cycling; recently, adipocytes were overlooked as niche cells that produce growth signals (46). In fact, intradermal adipocytes are recognized for more than just their roles in energy storage, thanks to their secretion of hormones, growth factors and cytokines, such as leptin, PDGF and tumor necrosis factor-alpha, as well as proteins related to immunological and vascular functions. Festa et al. (47), using histological and functional analysis of cell populations of the adipocyte lineage, analyzed the role of intradermal adipocytes on follicular stem cells activity. In this study they identified a dynamic process of adipogenesis that parallels the activation of hair follicle stem cells and revealed that immature adipocyte cells are necessary and sufficient to activate follicular stem cells. In particular, they focused on PDGFa secreted by adipocyte precursor cells as the factor to promote hair growth. In this view, we decided to treat patients with AGA for 6 months with application of adipose-derived stem cells (ADSCs) through the injection of adipose tissue lipoaspi- rates obtained from the abdomen and hip regions of donors using a cannula with small holes at a very superficial level of adipose tissue that allows a greater yield of adipose-derived stem cells (48). After 6 months, serial measurements indicated a significant increase in hair counts, without side effects. However, clinical trials are required to evaluate its long term efficacy and side effects.

\section{Surgical management}

In severe AGA, management option is hair transplantation surgery (18) which involves removal of hair follicles from the occipital scalp, which is relatively androgen resistant, and their reimplantation into the bald vertex and frontal scalp (4). Prerequisite for the procedure is sufficient hair on the occipital scalp; a scalp density of 100 follicular $\mathrm{U} / \mathrm{cm}^{2}$ is considered excellent (4). Follicular unit transplant (FUT) is considered the gold standard because it preserves the natural architecture of the hair units and gives natural results. Grafts for FUT may be obtained through the strip harvesting method or Follicular Unit Extraction (FUE) harvesting. In the strip harvest method, a strip of scalp is excised from the occipital scalp and the wound is sutured; the donor strip is then dissected under a microscope into the follicular units (FUTs) and then transplanted into the balding area (1).

In FUE harvesting, individual FUTs are extracted directly from the donor site with $1 \mathrm{~mm}$ punch biopsy and then, using a micro blade, each unit is reinserted back into the bald areas. Compared with the traditional strip harvest technique, FUE tends to be more costly and takes more time (48). More recently, automated FUE systems have been developed to reduce the procedure time and transection rate.

Surgical results can be adequately assessed after no less than 3 months because the transplanted follicle will take up to 3 months to reach the anagen phase and become noticeable and, in addition, transplanted hairs seem to immediately go into a telogen phase after insertion. There is always a degree of graft failure, depending on the skill of the surgeon, the density of graft placement, preparation of the graft units and their desiccation (1).

In patients in which the transplant is performed with the aim to increase the density of hairs in the front area or vertex, topical minoxidil and finasteride can be a useful adjunct therapy 
following hair transplantation in order to prevent non-transplanted hairs loss and in order to achieve their growth. Because of a different mechanism of action for each drug and the synergic effect when combined, it is recommended using both finasteride and minoxidil (4). The use of minoxidil in perioperative period has the advantage of stabilizing the hair loss, increasing the number of hair in anagen phase and decreasing post surgical telogen effluvium (49). Minoxidil should be stopped 2 to 3 days before surgery to reduce the risk of intraoperative bleeding caused by vasodilation; treatment should be restarted in 1-2 weeks (21). Randomized, double-blind trial using finasteride $1 \mathrm{mg}$ daily or placebo in 79 men with AGA 4 weeks before and 48 weeks after hair transplantation demonstrated that the treatment group had significant improvement from baseline, in comparison with placebo group (50)]. Another useful tool to support hair transplant surgery is the injection (subcutaneous, intradermal, interfollicular) of PRP in the scalp in order to encourage transplanted graft growth (39).

\section{Conclusions}

In conclusion, a better understanding of the pathophysiology of AGA should lead to more successful treatment based on multi-therapeutic approach, targeted at different pathophysiological aspects of AGA, such as excessive sensitivity of the hair follicle to androgen, microinflammation and PGs dysregulation. For this reason the treatment of AGA should be based on multiple drugs, considering also the pathological conditions associated, such as the inflammatory conditions of the scalp that are often encountered during medical examination and therapeutic expectations of patients. In the next future, the understanding of the molecular genetic etiology and pathophysiological aspects of AGA will provide a pathway to the development of more efficacious, personalized and targeted treatment options.

\section{References}

1. Rathnayake D, Sinclair R. Male androgenetic alopecia. Expert Opin Pharmacother 2010: 11 (8): 1295-1304.

2. Hamilton JB. Patterned hair loss in man: types and incidence. Ann NY Acad Sci 1951: 53 (3): 708-728.

3. Guarrera M, Rebora A. Anagen hairs may fail to replace telogen hairs in early androgenetic alopecia. Dermatology 1996: 192 (1): 28-31.
4. Rousso DE, Kim SW. A review of medical and surgical treatment options for androgenetic Alopecia. JAMA Facial Plast Surg 2014: 16 (6): 444-450.

5. Banka N, Bunagan MJ, Shapiro J. Pattern hair loss in men: diagnosis and medical treatment. Dermatol Clin 2013: 31 (1): 129-140.

6. Ellis JA, Sinclair RD. Male pattern baldness: current treatments, future prospects. Drug Discov Today 2008: 13 (1718): 791-797.

7. Ellis JA, Sinclair R, Harrap SB. Androgenetic alopecia: pathogenesis and potential for therapy. Expert Rev Mol Med 2002: 4 (22): 1-11.

8. Hillmer AM, Hanneken S, Ritzmann S., et al. Genetic variation in the human androgen receptor gene is the major determinant of common early-onset androgenetic alopecia. Am J Hum Genet 2005: 77 (1): 140-148.

9. Ellis JA, Harrap SB. The genetics of androgenetic alopecia. Clin Dermatol 2001: 19 (2): 149-154.

10. Randall VA. Androgens and human hair growth. Clin Endocrinol 1994: 40: 439-457.

11. Thornton MJ, Hamada K, Messenger AG, Randall VA. Beard, but not scalp, dermal papilla cells secrete autocrine growth factors in response to testosterone in vitro. J Invest Dermatol 1998: 111: 727-732.

12. Whiting DA. Diagnostic and predictive value of horizontal sections of scalp biopsy specimens in male pattern androgenetic alopecia. J Am Acad Dermatol 1993: 28 (5 Pt 1): 755-763.

13. Mahé YF, Michelet JF, Billoni N, et al. Androgenetic alopecia and microinflammation. Int J Dermatol 2000: 39 (8): 576-584.

14. Garza LA, Liu Y, Yang Z, et al. Prostaglandin D2 inhibits hair growth and is elevated in bald scalp of men with androgenetic alopecia. Sci Transl Med 2012: 4 (126): 126ra34-126ra21.

15. Johnstone MA, Albert DM. Prostaglandin-induced hair growth. Surv Ophthalmol 2002: 47 (Suppl 1): S185-S202.

16. Nieves A, Garza LA. Does prostaglandin D2 hold the cure to male pattern baldness? Exp Dermatol 2014: 23 (4): 224-227.

17. Blumeyer A, Tosti A, Messenger A, et al. Evidence-based (S3) guideline for the treatment of androgenetic alopecia in women and in men. J Dtsch Dermatol Ges 2011: 9 (Suppl 6): S1-S57.

18. Bienová $M$, Kucerová $R$, Fiurásková $M$, et al. Androgenetic alopecia and current methods of treatment. Acta Dermatovenerol Alp Pannonica Adriat 2005: 14 (1): 5-8.

19. Rossi A, Calvieri S. Treatment for Alopecia. G Ital Dermatol Venereol 2014: 149 (1): 103-106.

20. Lachgar S, Charveron M, Gall Y. Minoxidil upregulates the expression of vascular endothelial growth factor in human hair dermal papilla cells. Br J Dermatol 1998: 138 (3): 407-411.

21. Rossi A, Cantisani C, Melis L, Iorio A, Scali E, Calvieri S. Minoxidil use in dermatology, side effects and recent Patents. Recent Pat Inflamm Allergy Drug Discov 2012: 6 (2): 130-136.

22. Kaliyadan F, Nambiar A, Vijayaraghavan S. Androgenetic alopecia: an update. Indian J Dermatol Venereol Leprol 2013: 79: 613-625.

23. Gupta AK, Charrette A. Topical minoxidil: systematic review and Meta-analysis of its efficacy in androgenetic Alopecia. Skinmed 2015: 13 (3): 185-189.

24. Drake L, Hordinsky M, Fiedler V, et al. The effects of finasteride on scalp skin and serum androgen levels in men with androgenetic alopecia. J Am Acad Dermatol 1999: 41 (4): 550. 
25. Rossi A, Cantisani C, Scarnò M, et al. Finasteride, $1 \mathrm{mg}$ daily administration on male androgenetic alopecia in different age groups: 10-year follow-up. Dermatol Ther 2011: 24 (4): 455-461.

26. Rossi A, Carlesimo M, Pranteda G, et al. Finasteride for Androgenetic Alopecia and Side Effects 2013: 1 (4): 197-203.

27. Mysore V, Shashikumar BM. Guidelines on the use of finasteride in androgenetic alopecia. Indian J Dermatol Venereol Leprol 2016: 82 (2): 128-134.

28. Caserini M, Radicioni M, Leuratti C, Terragni E, Iorizzo M, Palmieri R. Effects of a novel finasteride $0.25 \%$ topical solution on scalp and serum dihydrotestosterone in healthy men with androgenetic alopecia. Int J Clin Pharmacol Ther 2016: 54 (1): 19-27.

29. Hajheydari Z, Akbari J, Saeedi M, Shokoohi L. Comparing the therapeutic effects of finasteride gel and tablet in treatment of the androgenetic alopecia. Indian J Dermatol Venereol Leprol 2009: 75 (1): 47-51.

30. Olsen EA, Hordinsky M, Whiting D, et al. The importance of dual 5alpha-reductase inhibition in the treatment of male pattern hair loss: results of a randomized placebocontrolled study of dutasteride versus finasteride. J Am Acad Dermatol 2006: 55 (6): 1014-1023.

31. Tsunemi Y, Irisawa R, Yoshiie H, et al. Long-term safety and efficacy of dutasteride in the treatment of male patients with androgenetic alopecia. J Dermatol 2016: 2: 1-8.

32. Prager N. A randomized double-blind placebo controlled trial to determine the effectiveness of botanically derived inhibitors of 5 alpha reductase in the treatment of androgenetic alopecia. J Altern Complent Med 2002: 8: 413-452.

33. Rossi A, Mari E, Scarno M, et al. Comparative effectiveness finasteride vs serenoa repens in male androgenetic alopecia: a two year study. Int J Immunopathol Pharmacol 2012: 25 (4): 1167-1173.

34. Piérard-Franchimont C, De Doncker P, Cauwenbergh G, Piérard GE. Ketoconazole shampoo: effect of long-term use in androgenic alopecia. Dermatology 1998: 196 (4): 474-477.

35. Keaney T. Emerging therapies for androgenetic Alopecia. J Drugs Dermatol 2015: 14 (9): 1036-1040.

36. Gali-Muhtasib H, Roessner A, Schneider-Stock R. Thymoquinone: a promising anti-cancer drug from natural sources. Int J Biochem Cell Biol 2006: 38 (8): 1249-1253.

37. Rossi A, Priolo L, Iorio A, et al. Evaluation of a therapeutic alternative for telogen effluvium: a pilot Study. J Cosm Dermatol Sci Appl 2013: 3: 9-16.
38. Arora NS, Ramanayake T, Ren YF, Romanos GE. Plateletrich plasma: a literature review. Implant Dent 2009: 18 (4): 303-310.

39. Uebel CO, da Silva JB, Cantarelli D, Martins P. The role of platelet plasma growth factors in male pattern baldness surgery. Plast Reconstr Surg 2006: 118 (6): 1458-1466.

40. Cervelli V, Garcovich S, Bielli A, et al. The effect of autologous activated platelet rich plasma (AA-PRP) injection on pattern hair loss: clinical and histomorphometric evaluation. Biomed Res Int 2014: 2014: 760709

41. Rangwala S, Rashid RM. Alopecia: a review of laser and light therapies. Dermatol Online J 2012: 18 (2): 3.

42. Kim H, Choi JW Kim JY, et al. Low-level light therapy for androgenetic alopecia: a 24-week, randomized, doubleblind, sham device-controlled multicenter trial. Dermatol Surg 2013: 39 (8): 1177-1183.

43. Jimenez JJ1, Wikramanayake TC, Bergfeld W, et al. Efficacy and safety of a low-level laser device in the treatment of male and female pattern hair loss: a multicenter, randomized, sham device-controlled, double-blind study. Am J Clin Dermatol 2014: 15 (2): 115-127.

44. Charlesworth EN, Kagey-Sobotka A, Norman PS, Lichtenstein LM. Effect of cetirizine on mast cellmediator release and cellular traffic during the cutaneous late-phase reaction. J Allergy Clin Immunol 1989: 83 (5): 905-912.

45. Roch-Arveiller M, Tissot M, Idohou N, et al. In vitro effect of cetirizine on PGE2 release by rat peritoneal macrophages and human monocytes. Agents Actions 1994: 43 (1-2): 13-16.

46. Schmidt B, Horsley V. Unravelling hair follicle-adipocyte communication. Exp Dermatol 2012: 21 (11): 827-830.

47. Festa E, Fretz J, Berry R, et al. Adipocyte lineage cells contribute to the skin stem cell niche to drive hair cycling. Cell 2011: 146 (5): 761-771.

48. Trivisonno A, Di Rocco G, Cannistra C, et al. Harvest of superficial layers of fat with a microcannula and isolation of adipose tissue-derived stromal and vascular cells. Aesthet Surg J 2014: 34 (4): 601-613.

49. Kassimir JJ. Use of topical minoxidil as a possible adjunct to hair transplant surgery.A pilot study. J Am Acad Dermatol 1987: 16: 685-687.

50. Leavitt M, Perez-Meza D, Rao NA, et al. Effects of finasteride (1 mg) on hair transplant. J Dermatol Surg 2005: 31: 1268-1276. 